\title{
Evaluación de la disponibilidad del hierro para la eritropoyesis en una población tratada por hemodiálisis crónica
}

\author{
María N. Campistrús-Forneiro ${ }^{1 *}$, Érica Ferrari-Gratulini², Juan C. Cazéres² ${ }^{2}$ Sandra Iglesias-Hernández¹, \\ Alicia Petraglia-Daglier ${ }^{1}$ y Teresa Gómez-Pérez ${ }^{1}$ \\ ${ }^{1}$ Centro de Diálisis Uruguayana; ${ }^{2}$ Laboratorio Fertilab. Uruguay
}

\section{Resumen}

En la hemodiálisis crónica (HDC), determinar el hierro disponible para la eritropoyesis implica algunos desafíos. La ferritina y la saturación de transferrina (SatT) tienen limitaciones. El porcentaje de eritrocitos hipocrómicos (\%Hypo) y contenido de hemoglobina reticulocitaria $(\mathrm{CHr})$ se consideran indicadores directos del hierro utilizable. Objetivo: Evaluar el hierro disponible mediante $\mathrm{CHr}$ y \%Hypo y analizar su relación con parámetros habituales, inflamación y respuesta a la eritropoyetina (Epo). Material y métodos: Estudio observacional y transversal en 80 pacientes con HDC, sin sangrado evidente, infección u operación reciente, neoplasia o talasemia; 64 pacientes recibían Epo. Se determinaron hemoglobina, \%Hypo, reticulocitos, CHr, sideremia, ferritina, transferrina y proteína C reactiva (PCR), expresados por mediana y percentiles 2.5 - 97.5 . Índice de resistencia a Epo $($ ResistEpo $)=($ dosis $/ \mathrm{kg} / \mathrm{sem}) /$ hemoglobina. Resultados: La población presentaba hemoglobina $=11.8 \mathrm{~g} / \mathrm{dl}$ (9.2-14.2), sideremia $=54.6 \mu \mathrm{g} / \mathrm{dl}(21-111)$, SatT $=21.3 \%$ (9.3-47.5), \%Hypo = 1.4\% (0.1-7.1), CHr $=32.4 \mathrm{pg}(27.5-35)$, ferritina $=422 \mathrm{ng} / \mathrm{ml}(26-1,396), P C R=9.5 \mathrm{mg} / \mathrm{L}$ (0.5-91.7). Sideremia y SatT se correlacionaron positivamente con \%Hypo y negativamente con $\mathrm{CHr}(p<0.001)$. SatT $\leq 20 \%$ y PCR $>10 \mathrm{mg} / \mathrm{L}$ determinaron el riesgo de \%Hypo > 3\%. Sideremia, SatT $\leq 20 \%$ y \% Hypo explicaron la ResistEpo > 12, desde \%Hypo >3\% (OR = 5.26; $\mathrm{p}=0.03)$. Conclusiones: $\% \mathrm{Hypo} \mathrm{y} \mathrm{CHr}$ complementan la evaluación del hierro en HDC. Se confirmó una relación entre inflamación y hierro disponible. \%Hypo > 3\% es el determinante de respuesta a Epo.

Palabras clave: Anemia. Hemodiálisis. \% de eritrocitos hipocrómicos. Hemoglobina reticulocitaria. Inflamación. Resistencia a eritropoyetina.

\section{Evaluation of iron availability for erythropoiesis in chronic hemodialysis patients}

\section{Abstract}

Estimating the iron available for erythropoiesis in chronic hemodialysis (CDH) involves challenges. Ferritin and transferrin saturation (SatT) have limitations. Percentage of Hypochromic Red Cells (\%Hypo) and Content of Reticulocyte Hemoglobin $(\mathrm{CHr})$ are considered direct indicators of usable iron. Objective: To evaluate iron availability using $\mathrm{CHr}$ and \%Hypo, analyzing its relationship with traditional parameters, inflammation and the response to erythropoietin (Epo). Patients and methods: Observational, cross-sectional study in 80 HDC patients, without evidence bleeding, infection, recent surgery, neoplasia nor thalassemia; 64 patients received Epo. Hemoglobin, \%Hypo, reticulocytes, CHr, serum iron, transferrin, ferritin and C-reactive protein (PCR) were expressed by median and 2.5-97.5\% percentiles. Epo resistance index

\section{Correspondencia:}

*María N. Campistrús-Forneiro E-mail: mncampist@gmail.com
Disponible en internet: 18-06-2021

Nefro Latinoam. 2021;18:50-57 www.nefrologialatinoamericana.com licencia CC BY-NC-ND (http://creativecommons.org/licenses/by-nc-nd/4.0/). 
$($ ResistEpo $)=($ weekly dose $/ \mathrm{kg}$ weight $) /$ hemoglobin. Results: Population presented: hemoglobin $=11.8 \mathrm{~g} / \mathrm{dl}$ (9.2-14.2), sideremia $=54.6 \propto \mathrm{g} / \mathrm{dl}(21-111)$, Sat $=21.3 \%(9.3-47.5), \% H y p o=1.4 \%(0.1-7.1), \mathrm{CHr}=32.4 \mathrm{pg}(27.5-35)$, ferritin $=422 \mathrm{ng} / \mathrm{ml}$ (26-1,396), $P C R=9.5 \mathrm{mg} / \mathrm{L}$ (0.5-91.7). Sideremia and SatT correlated positively with \%Hypo and negatively with $\mathrm{CHr}(\mathrm{p}<0.001)$. Sat $\mathrm{T} \leq 20 \%$ and $P C R>10 \mathrm{mg} / \mathrm{L}$ were independent determinants of $\% H y p o>3 \%$. Sideremia, Sat $\leq 20 \%$ and $\% H y p o$ explained ResistEpo $>12$, effect observed from \%Hypo $>3 \%(\mathrm{OR}=5.26 ; p=0.03)$. Conclusions: \%Hypo and $\mathrm{CHr}$ complement the iron assessment in HDC. Effect of inflammation on iron availability is confirmed. \%Hypo was determinant of Epo response at lower level than previously proposed.

Key words: Anemia. Hemodialysis. Percentage of hypochromic erythrocytes. Reticulocyte hemoglobin content. Erythropoietin resistance. Inflammation.

\section{Introducción}

La anemia es una complicación muy frecuente de la enfermedad renal crónica (ERC), en especial entre pacientes que requieren tratamiento sustitutivo por hemodiálisis crónica $(\mathrm{HDC})^{1,2}$. Una encuesta realizada en cinco países europeos mostró que $87 \%$ de los pacientes en diálisis presentaba anemia, definida por una hemoglobina $<12 \mathrm{~g} / \mathrm{dl}$ en mujeres $\mathrm{y}<13 \mathrm{~g} / \mathrm{dl}$ en varones, mientras que $78 \%$ recibía tratamiento con eritropoyetina 0 hierro $\mathrm{IV}^{3}$. En Latinoamérica se ha comprobado hemoglobina $(\mathrm{Hb})<10.5 \mathrm{~g} / \mathrm{dl}$ en $46 \%$ de los pacientes en HDC, aun cuando $83 \%$ de la población recibía agentes estimulantes de la eritropoyesis y $68 \%$ hierro intravenoso. Este estudio mostró asimismo que el índice de saturación de transferrina $<25 \%$ era un factor determinante independiente del riesgo de presentar anemia grave, caracterizada por $\mathrm{Hb}<10.5 \mathrm{~g} /$ $\mathrm{dl}^{4}$. Estudios anteriores, como el National Health and Nutritional Examination Survey (2004), encontraron alta prevalencia de carencia férrica, del orden de $58 \%$ en hombres y $72 \%$ en mujeres con ERC, aún sin hallarse en $\mathrm{HDC}^{5}$. En la población en HDC, con base en datos de 12 países, el Dialysis Outcomes and Practice Pattern Study (DOPPS) detectó carencia férrica, definida por saturación de transferrina $<20 \%$ en $35 \%$ a $40 \%$ de los pacientes estudiados ${ }^{6}$.

El tratamiento de la anemia en ERC requiere un adecuado balance entre el estímulo para la generación de eritroblastos, dependiente de la eritropoyetina, y el aporte de hierro suficiente para la síntesis de hemoglobina, por lo cual resulta fundamental evaluar el metabolismo férrico. No obstante, la cantidad óptima de hierro en estos pacientes aún persiste poco clara debido a las alteraciones de su homeostasis en ERC. Muchos pacientes portadores de ERC, en particular si se tratan con HDC, presentan balance negativo de hierro como consecuencia de restricciones dietéticas, reducción de su absorción y aumento de las pérdidas. No obstante, lo que se observa con más frecuencia en la HDC es la carencia funcional de hierro, caracterizada por depósitos de hierro adecuados (ferritina $>100 \mathrm{ng} / \mathrm{ml}$ ) pero poco eficaces para la síntesis de hemoglobina. Mientras el hierro de depósito se calcula con la ferritina sérica, habitualmente se usa el índice de saturación de transferrina para evaluar su disponibilidad. Múltiples factores influyen en ambos parámetros, en particular por la inflamación, por lo que resultan poco sensibles y específicos ${ }^{7-9}$. En consecuencia, se han buscado nuevos indicadores del metabolismo férrico, más exactos y menos dependientes del estado inflamatorio subyacente de la población con ERC en diálisis. La incorporación de nuevos analizadores hematimétricos para la práctica clínica, con tecnología basada en citometría de flujo, permite determinar el contenido de hemoglobina de reticulocitos $(\mathrm{CHr})$ y el porcentaje de eritrocitos hipocrómicos (\%Hypo), ambos marcadores directos del hierro utilizable por la médula ósea ${ }^{9-11}$. Por otra parte, dado que la respuesta al tratamiento con eritropoyetina (Epo) se afecta por la carencia férrica, así como por el estado inflamatorio, es razonable presuponer que exista una relación entre los nuevos parámetros y la resistencia a la $\mathrm{Epo}^{12}$.

\section{Objetivos}

Evaluar la disponibilidad del hierro para la eritropoyesis en pacientes en HDC mediante el contenido de $\mathrm{Hb}$ reticulocitaria y el porcentaje de GR hipocrómicos; analizar su relación con los parámetros usados con regularidad; e identificar su relación final con la inflamación y la respuesta al tratamiento con eritropoyetina (Epo).

\section{Población y métodos}

Se realizó un estudio observacional de corte transversal en un centro de hemodiálisis ambulatoria de Montevideo, Uruguay. Se seleccionó a pacientes con un mínimo de tres meses en HDC, sin proceso 
infeccioso ni sangrado evidente u operación durante los últimos tres meses. Se excluyó a los pacientes con neoplasia terminal y portadores de talasemia. Se incluyó a 80 pacientes, con edad promedio de $62 \pm 14$ años, 47 de los cuales $(59 \%)$ eran varones. La causa de la ERC se catalogó como glomerulopatía crónica en 21 pacientes $(26.3 \%)$, diabetes en $17(21.3 \%)$, nefropatía tubulointersticial en 22 pacientes $(27.5 \%)$, causa vascular en 17 pacientes (21.3\%) y no quedó determinada en $3(3.8 \%)$. La mediana del tiempo en diálisis fue de 31.5 meses (intervalo, 3-236). Ningún paciente recibió transfusión de sangre en los tres meses anteriores al estudio. Las sesiones de diálisis duraban, en promedio, 11.7 horas por semana $( \pm 0.7)$.

Se recogieron muestras de $5 \mathrm{ml}$ de sangre antes de comenzar la diálisis de mitad de semana y se enviaron al laboratorio de inmediato de modo que se procesaron antes de cuatro horas de extraídas.

Se determinaron hemograma, \%Hypo, reticulocitos, $\mathrm{CHr}$, sideremia, transferrina, ferritina sérica y proteína $C$ reactiva ( $P C R$ ). El hemograma, que incluye entre sus índices hematimétricos el $\mathrm{CHr}$, el \%Hypo y el recuento de reticulocitos, se calculó en un analizador automatizado ADVIA 2120 de Siemens. La sideremia se midió mediante método colorimétrico, la transferrina y la PCR se determinaron por ensayo inmunoturbidimétrico, la ferritina se midió por método inmunoenzimático, todo lo cual se analizó en el Dimension clinical chemistry system de Siemens. Se calculó el índice de saturación de transferrina $($ SatT $)=($ sideremia. 100$) /($ transferrina.1.4) $)^{13}$. La respuesta a la eritropoyetina se estableció con base en el índice de resistencia a Epo (ResistEpo) con la fórmula: (dosis semanal de Epo/kg de peso)/ hemoglobina ${ }^{14}$.

Análisis estadístico. Excepto la hemoglobina, que por su distribución normal se expresa por promedio y desviación estándar, los demás datos hematimétricos y bioquímicos presentaron una distribución anormal en esta población, por lo que se expresaron por su mediana y percentiles 2.5 - 97.5. Se aplicó la prueba de chi cuadrado para evaluar la relación entre variables categóricas. Se utilizó la prueba de "t" para comparar la distribución de hemoglobina entre grupos de pacientes y se usaron pruebas no paramétricas para comparaciones ( $U$ de Mann-Whitney) y correlaciones (coeficiente Rho de correlación de Spearman) entre los demás parámetros. Se aplicó el análisis por regresión logística binaria con el fin de evaluar la fortaleza de las relaciones entre los parámetros estudiados e identificar cuáles resultaban factores determinantes independientes, ya sea de la disponibilidad de hierro para la
Tabla 1. Parámetros hematimétricos y bioquímicos en la población total $(n=80)$

\begin{tabular}{|l|c|c|}
\hline Parámetros & Mediana & Percentiles $2.5^{\circ}-97.5^{\circ}$ \\
\hline Reticulocitos (Cel/McL) & 69,650 & $23,613-137,022$ \\
\hline$\% H y p o(\%)$ & 1.4 & $0.1-7.7$ \\
\hline CHr (pg) & 32.4 & $26.3-35.2$ \\
\hline Sideremia (ug/dl) & 54.6 & $16.5-114.2$ \\
\hline Transferrina (mg/dl) & 167 & $117.2-256$ \\
\hline Sat.T (\%) & 21.3 & $9.3-47.5$ \\
\hline Ferritina (ng/ml) & 422 & $22.0-1,406$ \\
\hline PCR (mg/L) & 9.6 & $0.4-140.6$ \\
\hline
\end{tabular}

\%Hypo: porcentaje de eritrocitos hipocrómicos; $\mathrm{CHr}$ : contenido de hemoglobina de reticulocitos; Sat.T: índice de saturación de transferrina; PCR: proteína C reactiva.

eritropoyesis o de la respuesta al tratamiento con Epo ${ }^{15}$. Se utilizó SPSS versión 25. Se exigió un nivel de significación estadística $<0.05$.

Ética. El protocolo del estudio se diseñó con apego a la Declaración de Helsinki y lo autorizó el comité de ética institucional (Fertilab-CEM). Las muestras se recogieron según el protocolo de control periódico habitual del centro de diálisis. Se obtuvo consentimiento de los pacientes participantes para utilizar sus datos y se garantizó su anonimato.

\section{Resultados}

La población presenta, en su conjunto, $\mathrm{Hb}$ promedio $=11.7 \pm 1.3 \mathrm{~g} / \mathrm{dl}$. Los demás parámetros hematimétricos y bioquímicos se resumen en la Tabla 1.

Se identificó a 37 pacientes con SatT $\leq 20 \%, 9$ de los cuales tenían ferritina $<200 \mathrm{ng} / \mathrm{ml}$. En la Tabla 2 se comparan los datos según fuera que la SatT resultara $>20 \% 0 \leq 20 \%$. El grupo con SatT $\leq 20 \%$ presentó $\mathrm{CHr}$ menor y \%Hypo más elevado que los pacientes con SatT $>20 \%$. No existió diferencia en la $\mathrm{Hb}$ entre esos grupos, pero la reticulocitosis es mayor en pacientes con SatT $\leq 20 \%$. La ferritina, así como la PCR, no muestran diferencia significativa. Por otra parte, la dosis de Epo de los pacientes tratados es mayor entre quienes presentan SatT $\leq 20 \%: 119 \mathrm{UI} / \mathrm{kg} / \mathrm{sem}(14-269)$ contra $61 \mathrm{UI} / \mathrm{kg} / \mathrm{sem}$ (10-221), $\mathrm{p}=0.02$.

\section{Correlaciones entre parámetros de la población}

total. $\mathrm{La} \mathrm{Hb}$ se correlaciona positivamente con la sideremia (Coef. $=0.243, p=0.03$ ) y negativamente con ferritina (Coef. $=-0.344, p=0.002$ ) y PCR (Coef. $=-0.224$, $\mathrm{p}=0.046)$. CHr y \%Hypo se correlacionan entre sí de 
Tabla 2. Comparación de parámetros de la población según el índice de saturación de transferrina

\begin{tabular}{|c|c|c|c|}
\hline Variables & SatT $\leq 20 \%$ & SatT $>20 \%$ & $\mathbf{p}$ \\
\hline $\begin{array}{l}\text { Número de } \\
\text { pacientes }\end{array}$ & 37 & 43 & \\
\hline Hemoglobina & $\begin{array}{l}11.6 \mathrm{~g} / \mathrm{dl} \\
(8.9-14.3)\end{array}$ & $\begin{array}{l}12.1 \mathrm{~g} / \mathrm{dl} \\
(9.9-13.7)\end{array}$ & 0.18 \\
\hline \%Нуро & $\begin{array}{c}2.2 \% \\
(0.3-7.9)\end{array}$ & $\begin{array}{c}0.6 \% \\
(0.1-5.1)\end{array}$ & $<0.001$ \\
\hline $\mathrm{CHr}$ & $\begin{array}{c}30.5 \mathrm{pg} \\
(26.3-34.9)\end{array}$ & $\begin{array}{c}33.2 \mathrm{pg} \\
(30.2-35.2)\end{array}$ & $<0.001$ \\
\hline Reticulocitos VA & $\begin{array}{c}77,900 \\
(47,380-142,860)\end{array}$ & $\begin{array}{c}67,300 \\
(23,700-117,220)\end{array}$ & 0.02 \\
\hline Sideremia & $\begin{array}{c}34.1 \mathrm{ug} / \mathrm{dl} \\
(15.76-62.7)\end{array}$ & $\begin{array}{l}73.0 \mathrm{ug} / \mathrm{dl} \\
(43.6-114)\end{array}$ & $<0.001$ \\
\hline Ferritina & $\begin{array}{l}380 \mathrm{ng} / \mathrm{ml} \\
(13-1,367)\end{array}$ & $\begin{array}{c}440 \mathrm{ng} / \mathrm{ml} \\
(152-1,406)\end{array}$ & 0.06 \\
\hline PCR & $\begin{array}{c}9.7 \mathrm{mg} / \mathrm{L} \\
(0.95-141.5)\end{array}$ & $\begin{array}{l}9.0 \mathrm{mg} / \mathrm{L} \\
(0.4-33.6)\end{array}$ & 0.09 \\
\hline
\end{tabular}

los datos se expresan por mediana y percentiles $2.5^{\circ}-97.5^{\circ}$. Comparación realizada por prueba $\mathrm{U}$ de Mann-Whitney. Abreviaturas: \%Hypo: porcentaje de eritrocitos hipocrómicos; $\mathrm{CHr}$ : contenido de hemoglobina de reticulocitos.

PCR: proteína C reactiva. SatT: índice de saturación de transferrina. VA: valor absoluto.

forma negativa (Coef. $=-0.588, p<0.001$ ). Sideremia y SatT se correlacionan de modo negativo con \%Hypo (Coef. $=-0.504, p<0.001$; Coef. $=-0.473, p<0.001$, respectivamente) y de modo positivo con $\mathrm{CHr}$ (Coef. $=0.593, p<0.001$; Coef. $=0.612, p<0.001$, respectivamente). La ferritina se correlaciona con $\mathrm{CHr}$ (Coef. $=0.262, p=0.019$ ) y con SatT (Coef. $=0.231$, $\mathrm{p}=0.039)$ y negativamente con transferrina (Coef. $=-0.309, p=0.005$ ), sin evidenciar un nexo con $\% H y p o$ ni con sideremia. La reticulocitosis absoluta se correlaciona con \%Hypo positivamente (Coef. $=0.435$, $\mathrm{p}<0.001$ ) y negativamente con $\mathrm{CHr}$ (Coef. $=-0.254$, $p=0.023)$ y SatT (Coef. $=-0.243, p=0.03$ ). $\mathrm{PCR}$ se vincula positivamente con \%Hypo (Coef. $=0.416 . p<0.001)$ y negativamente con $\mathrm{CHr}$ (Coef. $=-0.29, p=0.009$ ), sideremia (Coef. $=-0.357$, $p=0.001$ ), transferrina (Coef. $=-0.351, p=0.001$ ) y SatT (Coef. $=-0.25, p=0.025$ ). No se evidencia su relación con la ferritina sérica. La Figura 1A-D muestra algunas de estas vinculaciones.

Las correlaciones observadas sugieren aplicar el modelo de regresión logística para identificar cuáles de las variables pueden explicar, de modo independiente, una disponibilidad insuficiente de hierro para la eritropoyesis, medida de forma directa por \%Hypo elevado o $\mathrm{CHr}$ bajo. Se define \%Hypo $>3$ y $\leq 3$ como variable
Tabla 3. Factores explicativos independientes de baja disponibilidad de hierro para la eritropoyesis con base en el modelo de regresión logística

\begin{tabular}{|l|c|c|c|c|c|c|}
\hline Variables & B & ES & Wald & p & $\begin{array}{c}\text { Exp } \\
\text { (B) }\end{array}$ & $\begin{array}{c}\text { IC95\% } \\
\text { para } \\
\text { Exp (B) }\end{array}$ \\
\hline SatT $\leq 20 \%$ & 2.89 & 0.84 & 11.88 & 0.001 & 17.92 & $3.47-92.5$ \\
\hline $\begin{array}{l}\text { PCR } \\
>10 \mathrm{mg} / \mathrm{l}\end{array}$ & 1.72 & 0.69 & 6.29 & 0.01 & 5.61 & $1.46-21.6$ \\
\hline $\begin{array}{l}\text { Ferritina } \\
\leq 200 \mathrm{ng} / \mathrm{ml}\end{array}$ & -0.13 & 0.79 & 0.03 & 0.87 & 0.88 & $0.19-4.16$ \\
\hline Constante & -3.95 & 1.08 & 13.50 & $<0.01$ & 0.02 & \\
\hline
\end{tabular}

Variable dependiente: $\%$ Hypo $>3=1 ; \%$ Hypo $\leq 3=0$ (referencia).

Método "entrar". Porcentaje global pronosticado: $82.5 \%$. R: cuadrado de Nagelkerke $=0.42$. Abreviaturas. B: coeficiente de regresión (beta); ES: error estándar del coeficiente beta; Exp (B): exponencial del coeficiente beta; IC95\%: intervalo de confianza del 95\%; \%Hypo: porcentaje de eritrocitos hipocrómicos; SatT: índice de saturación de transferrina; PCR: proteína C reactiva.

dependiente y se explora su relación con los otros parámetros férricos relevantes y con PCR. La Tabla 3 muestra los resultados de dicho análisis, por el cual la posibilidad de que el \%Hypo sea superior a $3 \%$ se incrementa 17.9 veces si la Sat.T es $\leq 20 \%(p=0.001)$, así como ese riesgo aumenta 5.61 veces si la PCR es $>10 \mathrm{mg} / \mathrm{l}(\mathrm{p}=0.01)$.

Se aplica un análisis similar al $\mathrm{CHr}$, tras definir como variable dependiente binaria el $\mathrm{CHr}$ hasta 29 y $<29 \mathrm{pg}$ (referencia). La única variable independiente capaz de explicar valor bajo de $\mathrm{CHr}$ es la Sat.T $\leq 20 \%$ (OR $=16.9$, $p=0.019$; $R$ cuadrado de Nagelkerke $=0.37$; porcentaje global pronosticado $=92.5$ ), sin evidencia de efecto de otros indicadores férricos ni de la PCR.

Tratamiento de la anemia y su relación con marcadores férricos y de inflamación. Dosis de hierro intravenoso recibidas por la población en los últimos tres meses: se administran $100 \mathrm{mg}$ cada 2 meses a 13 pacientes $(16.3 \%), 32$ pacientes $(40 \%)$ recibieron $100 \mathrm{mg}$ por mes, 18 pacientes $(22.5 \%)$ recibieron $200 \mathrm{mg}$ por mes y 2 pacientes $(2.5 \%)$ recibieron $400 \mathrm{mg} / \mathrm{mes} ; 15$ pacientes no recibieron hierro (18.8\%).

No se observa diferencia en los valores de $\mathrm{Hb}$, SatT, ferritina, así como de \%Hypo y $\mathrm{CHr}$ sea que los pacientes reciban hierro intravenoso o no. No obstante, entre los 66 pacientes con ferritina $>200 \mathrm{ug} / \mathrm{l}, 57$ recibieron hierro intravenoso, mientras que sólo 8 de los 14 pacientes con ferritina $\leq 200 \mathrm{ug} / \mathrm{l}$ lo recibieron (ji cuadrada $=6.47, p=0.011$ ). En consecuencia, en esta población, el uso de FelV se vincula con ferritina $>200 \mathrm{ug} / \mathrm{l}$. No se halló un nexo con SatT >20\%. 

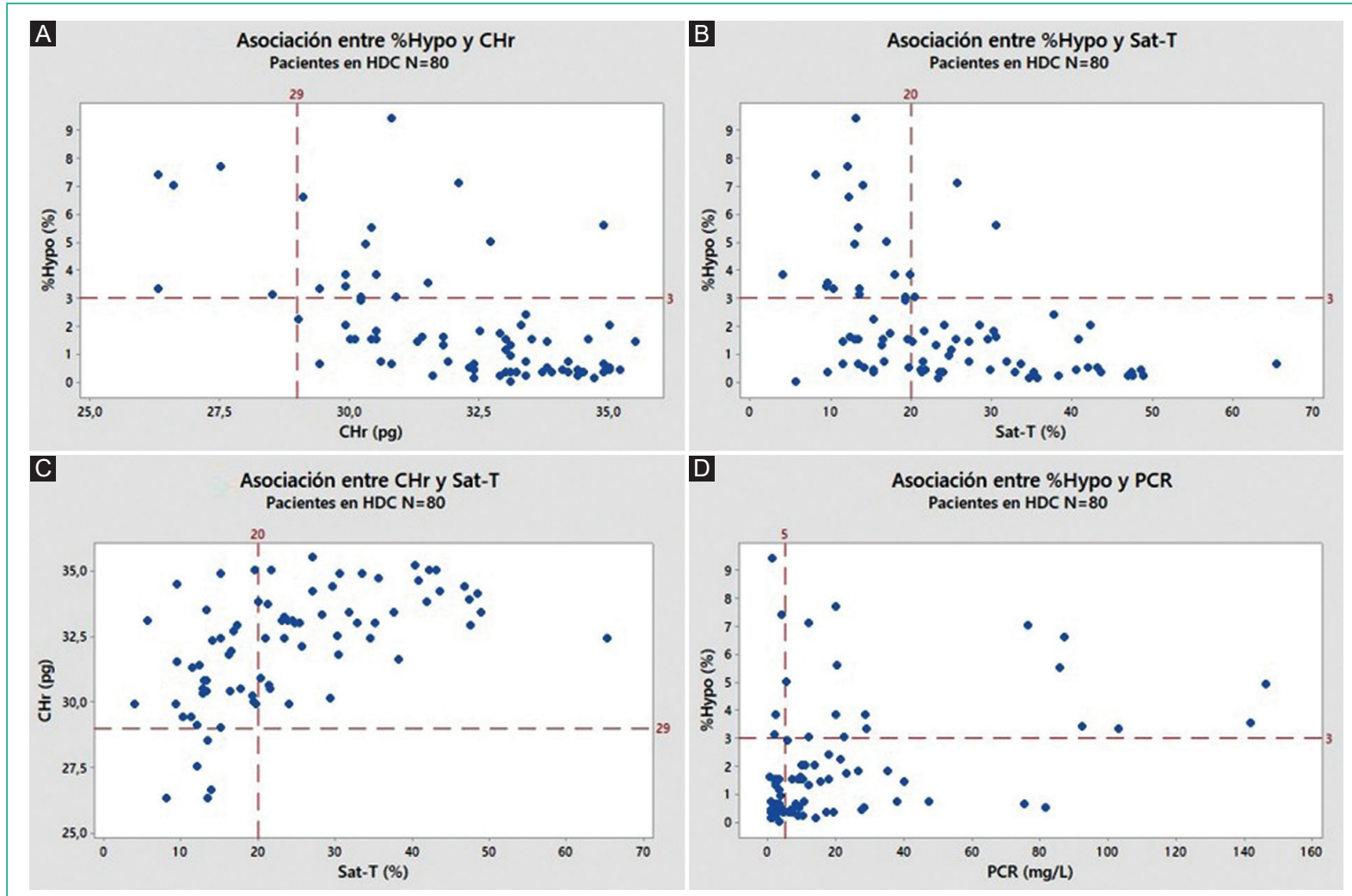

Figura 1. A: Relación entre el porcentaje de eritrocitos hipocrómicos (\%Hypo) y hemoglobina reticulocitaria (CHr). Las líneas de referencia marcan el punto de corte para \%Hypo $=3 \%$ y $\mathrm{CHr}=29 \mathrm{pg}$. B: Relación entre el \%Hypo y el porcentaje de saturación de la transferrina (Sat-T). Las líneas de referencia marcan el punto de corte para $\% \mathrm{Hypo}=3 \%$ y Sat-T $=20 \%$. C: Relación entre la CHr y el porcentaje de Sat-T. Las líneas de referencia muestran el punto de corte para $\mathrm{CHr}=29 \mathrm{pg}$ y Sat-T $=20 \%$. D: Relación entre el \%Hypo y la proteína $\mathrm{C}$ reactiva (PCR). Las líneas de referencia marcan el punto de corte para $\%$ Hypo $=3 \%$ y $P C R=5 \mathrm{mg} / \mathrm{L}$.

La dosis de Epo recibida por 64 pacientes, expresada por mediana y percentiles $2.5^{\circ}-97.5^{\circ}$, era $4,000 \mathrm{UI} / \mathrm{sem}$ (1,000-15,625), que correspondía a $75.4 \mathrm{Ul} / \mathrm{kg}$ peso/sem (11.9-257.9). El índice de resistencia a Epo calculado resultó $=6.9(1.1-24.4) \quad($ mediana $\mathrm{y}$ percentiles $\left.2.5^{\circ}-97.5^{\circ}\right)$.

Los pacientes con Epo presentaron $\mathrm{Hb}$ menor que aquéllos que no la recibían: $11.4 \pm 1.2 \mathrm{~g} / \mathrm{dl}$ contra 12.8 $\pm 1.1 \mathrm{~g} / \mathrm{dl}, \mathrm{p}<0.001$. La reticulocitosis no era diferente. Los únicos marcadores férricos diferentes, según fuera que se recibiera o no tratamiento con Epo, fueron la sideremia, menor entre los pacientes tratados (51.3 mg/dl, 20.4-102.1 contra $70.4 \mathrm{mg} / \mathrm{dl}$, 26.3115.3; $p=0.023)$, y la ferritina, más elevada en ese grupo (441 ng/ml, 97-1,390 contra $255 \mathrm{ng} / \mathrm{ml}, 13-868$; $p=0.019)$. Las dosis de hierro intravenoso administradas no eran diferentes según se recibiera o no Epo.

En la población tratada con Epo, la $\mathrm{Hb}$ se correlaciona positivamente con SatT (Coef. $=0.25, p=0.04$ ) y negativamente con PCR (Coef. $=-0.25, p=0.048$ ). Al igual que en la población total, sideremia y SatT de pacientes con Epo se correlacionan positivamente con $\mathrm{CHr}(\mathrm{p}<0.001)$ y negativamente con \%Hypo $(\mathrm{p}<0.001)$ y PCR $(p<0.02)$. PCR se vinculó con $\mathrm{CHr}$ (Coef. $=-0.36$, $p=0.003)$ y \%Hypo (Coef. $=0.47, p<0.001)$. El índice de resistencia a Epo se correlaciona con \%Hypo (Coef. $=0.48 ; p<0.001)$ y negativamente con sideremia (Coef. $=-0.40 ; p=0.001$ ) y SatT (Coef. $=-0.29$; $p=0.02)$. La Figura 2 muestra gráficamente estas relaciones.

Se analiza, por regresión logística, qué parámetros tienen mayor fortaleza como determinantes independientes de respuesta a Epo. ResistEpo se transforma en variable categórica: $>12(n=17) 0 \leq 12$ (referencia). Las únicas variables continuas capaces de explicar, de modo independiente, ResistEpo $>12$ son sideremia $(O R=0.94, p=0.019)$ y \% Hypo $(O R=1.62, p=0.012)$. 


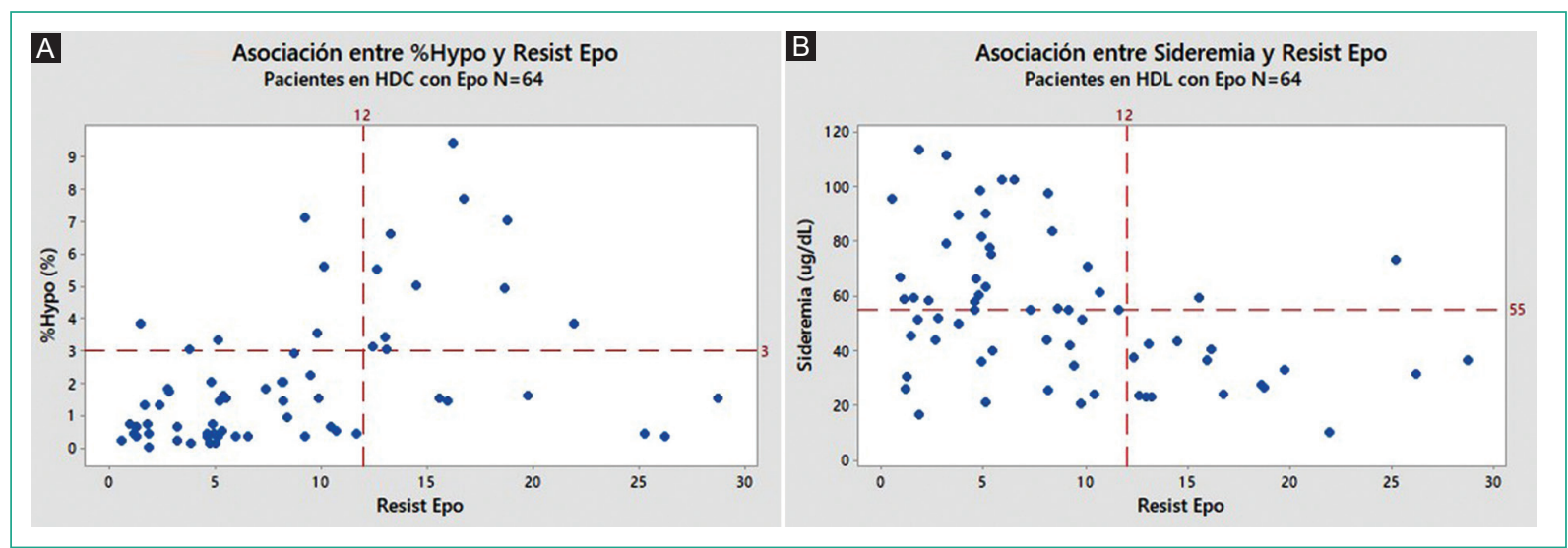

Figura 2. A: Relación entre el porcentaje de eritrocitos hipocrómicos (\%Hypo) y el índice de resistencia a la eritropoyetina (ResistEpo). Las líneas de referencia marcan el punto de corte para \%Hypo =3\% y ResistEpo = 12. B: Relación entre la sideremia y ResistEpo. Las líneas de referencia marcan el punto de corte para sideremia = 55 ug/dl y ResistEpo = 12 .

Tabla 4. Análisis de la relación entre indicadores de metabolismo férrico y el índice de resistencia a Epo por regresión logística binaria

\begin{tabular}{|l|c|c|c|c|c|c|}
\hline Variables & B & ES & Wald & p & $\begin{array}{c}\text { Exp } \\
\text { (B) }\end{array}$ & $\begin{array}{c}\text { IC95\% } \\
\text { para Exp } \\
\text { (B) }\end{array}$ \\
\hline $\begin{array}{l}\text { SatT } \\
\leq 20 \%\end{array}$ & 2.34 & 0.91 & 6.61 & 0.01 & 10.39 & $1.74-61.85$ \\
\hline $\begin{array}{l}\text { \%Hypo } \\
>3 \%\end{array}$ & 1.66 & 0.76 & 4.79 & 0.03 & 5.26 & $1.19-23.27$ \\
\hline $\begin{array}{l}\text { CHr } \\
\leq 29 \text { pg }\end{array}$ & 0.87 & 1.30 & 0.44 & 0.51 & 2.38 & $0.18-30.53$ \\
\hline $\begin{array}{l}\text { Ferritina } \\
\text { ng/ml) }\end{array}$ & 0.00 & 0.00 & 0.89 & 0.35 & 1.00 & $0.99-1.003$ \\
\hline \begin{tabular}{l} 
Constante \\
\hline
\end{tabular} & -3.57 & 1.04 & 11.76 & $<0.01$ & 0.03 & \\
\hline
\end{tabular}

Variable dependiente: ResistEpo $>12$; referencia: ResistEpo $\leq 12$.

Método: entrar. Porcentaje global: $82.8 \%$; R: cuadrado de Nagelkerke $=0.46$.

Abreviaturas. B: coeficiente de regresión (beta); ES: error estándar del coeficiente beta; $\operatorname{Exp}(\mathrm{B})$ : exponencial del coeficiente beta; IC95\%: intervalo de confianza de 95\%; \%Hypo: porcentaje de eritrocitos hipocrómicos; $\mathrm{CHr}$ : contenido de

hemoglobina reticulocitaria; ResistEpo: índice de resistencia a eritropoyetina.

La Tabla 4 muestra, categorizadas, las variables independientes de acuerdo con criterios clínicos, que así como la Sat $\mathrm{T} \leq 20 \%$ es determinante independiente de la respuesta a Epo, el \%Hypo es capaz de ejercer también ese efecto desde un valor $>3 \%$. De tal modo que, según los datos recogidos, un paciente en HDC tratado con Epo que presente \%Hypo >3\% tiene una probabilidad 5.3 veces mayor de desarrollar índice de resistencia a Epo $>12$ que si su \%Hypo fuera $\leq 3 \%$.

\section{Discusión}

La importancia de la determinación de \%Hypo y $\mathrm{CHr}$, en particular en pacientes con ERC, se ha demostrado hace más de 20 años, dadas sus elevadas especificidad, sensibilidad y capacidad para controlar el tratamiento ${ }^{16,17}$. El estudio no pretende más que aplicar esos parámetros en una población en HDC de Uruguay, donde la tecnología para su medida no ha estado disponible hasta hace poco tiempo. Se analizó a una población en HDC clínicamente estable, con adecuado control de la anemia, en la cual el $80 \%$ de los pacientes recibía tratamiento con Epo o hierro intravenoso.

De acuerdo con los criterios habitualmente aceptados por las guías de práctica clínica, 37 de los 80 pacientes presentaban signos de carencia férrica que, para la gran mayoría, se caracterizaría como carencia funcional $7,18,19$. Este grupo no evidencia anemia más grave, pero requiere mayor dosis de Epo para su control. Su \%Hypo es más elevado y su $\mathrm{CHr}$ menor que el de los pacientes con SatT $>20 \%$. Sin embargo, en la Figura 1B-C se observa marcada dispersión de estos indicadores entre los pacientes con supuesta "carencia férrica". Por otra parte, si bien las correlaciones entre sideremia y SatT con los nuevos parámetros son significativas, los coeficientes no resultan muy elevados, lo que se explicaría por la menor sensibilidad y especificidad reconocidas para aquéllas en relación con la observada para \%Hypo y $\mathrm{CHr}^{20-23}$. Se ha mostrado también la ausencia de relación significativa entre $\%$ Hypo y ferritina, algo que ya mostraron otros autores. 
Asimismo, se ha demostrado que la PCR es un factor explicativo independiente del nivel de \%Hypo, lo que indica cuánto influye la inflamación sobre la disponibilidad del hierro para la síntesis de hemoglobina. Éste sería un motivo por el cual el valor de ferritina, que es marcador de fase aguda de la inflamación, pierde confiabilidad como indicador aislado del metabolismo férrico en HDC. Se reconoce que en esta situación la homeostasis del hierro está alterada, ya que depende de la hepcidina, que inhibe tanto la absorción intestinal de hierro como su liberación de los macrófagos. La acción de la hepcidina está acentuada en la ERC porque su eliminación renal disminuye así como aumenta su síntesis hepática por el incremento de citocinas inflamatorias ${ }^{1,8,11,22,23}$.

De acuerdo con los datos, el \%Hypo $>3 \%$ permite explicar mayor resistencia al tratamiento con Epo, valor muy inferior al considerado "umbral" para pacientes en HDC por múltiples publicaciones y guías de práctica clínica 9,19,22,24. Dicho "umbral", definido como \%Hypo $>6 \%$, no representa un dato de normalidad, sino que se ha establecido por su excelente capacidad de predecir adecuada respuesta hematológica al tratamiento con hierro intravenoso ${ }^{7},{ }^{21}$. Bovy, et al. midieron \%Hypo en 537 donantes de sangre sanos y hallaron valores entre 0 y $3.7 \%$. En esa publicación se estudió a una población de 64 pacientes en HDC y se observó, además de buenas correlaciones con otros marcadores férricos, que el \%Hypo se correlacionaba con la dosis recibida de Epo $(p<0.01)$. Todos sus pacientes con SatT $\geq 30 \%$ o sideremia $>12 \mathrm{mmol} / \mathrm{L}$ presentaron $\%$ Hypo $<3.7 \%$, por lo que éste podría considerarse el límite superior del valor "normal" según ese estudio ${ }^{25}$.

El valor de Hypo $>6 \%$ se ha avalado en los estudios citados, dada su elevada sensibilidad para predecir respuesta al aporte de hierro IV; no obstante, podría resultar inadecuado para detectar situaciones de eritropoyesis ferropénica incipiente, como probablemente ocurre en esta población, la cual a partir de \%Hypo $>3 \%$ necesita mayor dosis de Epo para lograr un valor similar de $\mathrm{Hb}$. Esta hipótesis debería comprobarse con un estudio longitudinal y controlado, tal y como lo han realizado antes otros especialistas ${ }^{26}$.

Los nuevos marcadores de la homeostasis del hierro poseen, además de mayor sensibilidad y especificidad ya comentadas, el beneficio relacionado con su mínimo costo, ya que los analizadores hematimétricos que realizan su determinación usan la misma muestra extraída para hemograma y recuento reticulocitario sin costo adicional. Según una encuesta entre nefrólogos de Latinoamérica, sólo $37 \%$ de ellos refirió no enfrentar limitaciones para evaluar el capital férrico de sus pacientes en $\mathrm{HDC}^{27}$. Por consiguiente, al disponer del equipamiento apropiado, la determinación de \%Hypo y $\mathrm{HCr}$ facilitaría y economizaría dicho estudio.

Se destaca, no obstante, que existen limitaciones metodológicas a considerar. Es obligatorio asegurar un período máximo de seis horas entre la extracción de la muestra y su procesamiento para que \%Hypo y $\mathrm{CHr}$ resulten confiables. Asimismo, estos marcadores no sirven como indicadores de hierro disponible para la eritropoyesis en presencia de talasemia, en cuyo caso debe descartarse ese diagnóstico en pacientes de riesgo. La talasemia menor es la hemoglobinopatía más prevalente en el medio, evidenciable al observar microcitosis con aumento del recuento de eritrocitos, indicador de una médula compensatoria muy activa, que se confirma por electroforesis de la hemoglobina. Por otra parte, dada la breve persistencia de los reticulocitos circulantes, el CHr sólo es capaz de calcular la biodisponibilidad reciente de hierro, al identificar cambios de su homeostasis ocurridos en los últimos cinco a seis días. En cambio, dada su permanencia prolongada en la circulación, la determinación del \%Hypo se relaciona con la disponibilidad del hierro de los últimos dos a tres meses y por ello se ha reconocido como un indicador de eritropoyesis ferropénica subaguda y crónica ${ }^{10,11,20}$

\section{Conclusiones}

Estos datos permiten concluir, dada la buena relación entre parámetros tradicionales y los nuevos, que $\% \mathrm{Hypo}$ y $\mathrm{CHr}$ complementan adecuadamente la evaluación del hierro disponible en HDC, de modo práctico y económico. El \%Hypo se vincula de manera independiente con la PCR, lo que señala la importancia clínica de la inflamación como factor regulador de la disponibilidad del hierro en ERC. Se demostró asimismo que la sideremia, la SatT y el \%Hypo son factores determinantes independientes de la respuesta a Epo, y que este efecto se observa desde \%Hypo $>3 \%$, valor menor al considerado "umbral" por las guías de práctica clínica vigentes.

Para evaluar el metabolismo férrico en ERC se enfrentan dos problemas principales: a) la escasa sensibilidad y especificidad de los indicadores habitualmente usados y b) la influencia del estado inflamatorio sobre la cinética del hierro. Dado que la presencia de hierro en los eritrocitos es el aspecto más importante de su metabolismo, es fundamental contar con pruebas confiables capaces de medirlo. La CHr y el \%Hypo han demostrado ser útiles para evaluar la disponibilidad del 
hierro para la eritropoyesis. Se sugiere además que, en virtud de su mínimo costo, su determinación debe recomendarse en la práctica clínica.

\section{Financiamiento}

La presente investigación no ha recibido ayudas específicas provenientes de agencias del sector público, sector comercial o entidades sin ánimo de lucro.

\section{Conflicto de intereses}

Los autores declaran no tener conflicto de intereses.

\section{Agradecimientos}

Este estudio ha sido posible gracias a la colaboración de:

- La Dirección del Laboratorio Fertilab y sus licenciados en análisis clínicos Mario Figueroa, Blanca Ceria, María Rubini, Adriana Corbesola, Santiago Ruiz y Macarena Suárez.

- Los técnicos y licenciados de enfermería del Centro de Diálisis Uruguayana.

\section{Responsabilidades éticas}

Protección de personas y animales. Los autores declaran que los procedimientos seguidos se conformaron a las normas éticas del comité de experimentación humana responsable y de acuerdo con la Asociación Médica Mundial y la Declaración de Helsinki.

Confidencialidad de los datos. Los autores declaran que han seguido los protocolos de su centro de trabajo sobre la publicación de datos de pacientes.

Derecho a la privacidad y consentimiento informado. Los autores han obtenido el consentimiento informado de los pacientes o sujetos referidos en el artículo. Este documento obra en poder del autor de correspondencia.

\section{Bibliografía}

1. Fishbane S, Spinowitz B. Update on anemia in ESRD and earlier stages of CKD: Core Curriculum 2018. Am J Kidney Dis. 2018;71(3):423-435.

2. Stauffer ME, Fan T. Prevalence of anemia in chronic kidney disease in the United States. PLoS ONE 2004;9(1): e84943. doi:10.1371/journal. pone.0084943.

3. Eriksson D, Goldsmith D, Teitsson S, Jackson J, Nooten F. Cross-sectional survey in CKD patients across Europe describing the association between quality of life and anaemia. BMC Nephrology 2016;17:97. DOI 10.1186/s12882-016-0312-9. Consultado el 17/10/2020.

4. Carlini R, Obrador G, Campistrús MN, Andrade L, Chifflet L, Bregman R, et al. The first report of the Latin American Society of Nephrology and Hypertension (SLANH) Anemia Committee in Chronic Hemodialysis Patients. Nefrología. 2014;34(1):96-104.
5. Fishbane S, Pollack S, Feldman HI, Joffe MM. Iron indices in chronic kidney disease in the National Health and Nutritional Examination Survey 1988-2004. Clin J Am Soc Nephrol 2009;4:57-61. doi: 10.2215/ CJN.01670408.

6. Pisoni RL, Bragg-Gresham JL, Young EW, Mendelssohn DC, Held PJ, Port FK, et al. Anemia management and outcomes from 12 countries in the Dialysis Outcomes and Practice Patterns Study (DOPPS). Am J Kidney Dis. 2004;44:94-111.

7. Macdougall IC, Bircher AJ, Eckardt K-U, Obrador GT, Pollock CA, Stenvinkel $\mathrm{P}$, et al. Iron management in chronic kidney disease: conclusions from a "Kidney Disease: Improving Global Outcomes" (KDIGO) Controversies Conference. Kidney International. 2016;89:28-39.

8. Gafter-Gvili A, Schechter A, Rozen-Zvi B. Iron deficiency anemia in chronic kidney disease. Acta Haematol 2019;142:44-50.

9. Gaweda A. Markers of iron status in chronic kidney disease. Hemodialysis International 2017;21:S21-S27.

10. Urrechaga E, Borque L, Escanero JF. Biomarkers of hypochromia: the contemporary assessment of iron status and erythropoiesis. BioMed Research International 2013, Article ID 603786:1- 8. http://dx.doi. org/10.1155/2013/603786

11. Hayes W. Measurement of iron status in chronic kidney disease. Pediatric Nephrology. 2019:34:605-613. https://doi.org/10.1007/s00467-018-3955-x

12. Gillespie IA, Macdougall IC, Richards S, Jones V, Marcelli D, Froissart M, et al. Factors precipitating erythropoiesis-stimulating agent responsiveness in a european haemodialysis cohort: case-crossover study. Pharmacoepidemiology and Drug Safety. 2015;24:414-425. doi: 10.1002/pds.

13. Pérez-Surribas D, Gella-Concustella A, Cruz-Iglesias E, Hermoso-Durán S, Urrechaga-Igartua E, Alcaide-Martín MJ, et al. Estudio de la ferropenia en el laboratorio clínico. Rev Lab Clin. 2019;12(4):e34-e53. https://doi.org/10.1016/j.labcli.2019.01.004.

14. López-Gómez JM, Portolés JM, Aljama P. Factors that condition the response to erythropoietin in patients on hemodialysis and their relation to mortality. Kidney International 2008;74(Suppl 111):S75-S81.

15. Fiuza Pérez MD, Rodríguez Pérez JC. La regresión logística: una herramienta versátil. Nefrología. 2000;20(6):477-565; Consultado el 26/8/2020 en: www.revistanefrologia.com

16. Schaefer R, Schaefer L. Evaluation of iron status in renal failure patients. Hypochromic red blood cells and reticulocytes. Kidney International. 1999;55(Suppl. 69):S-44-S-48.

17. Braun J, Lindner K, Schreiber M, Heidler RA, Horl WH. Percentage of hypochromic red blood cells as predictor of erythropoietic and iron response after IV iron supplementation in maintenance haemodialysis patients. Nephrol Dial Transplant. 1997;12:1173-1181.

18. Carlini RG, Campistrús MN, Andrade L, Blanco C, Chifflet L, Cruz ZC, et al. Recomendaciones para el estudio y tratamiento de la anemia en pacientes con enfermedad renal crónica. Actualización mayo 2017. Nefrol Latinoam. 2017;14(3):85-116.

19. Revised european best practice guidelines for the management of anaemia in patients with chronic renal failure. Appendix B: assessment of iron stores and functional iron deficiency. Nephrol Dial Transplant. 2004;19 [Suppl 2]: ii39-ii41.

20. Buttarello M, Pajola R, Novello E, Rebeschini M, Cantaro S, Oliosi $\mathrm{F}$, et al. Diagnosis of iron deficiency in patients undergoing hemodialysis. Am J Clin Pathol. 2010;133:949-954. DOI: 10.1309/AJCPQAXOJFHFSOOA

21. Chung M, Moorthy D, Hadar N, Salvi $P$, lovin RC, Lau J. Biomarkers for assessing and managing iron deficiency anemia in late-stage chronic kidney disease. Comparative effectiveness. Review No. 83. AHRQ Publication No. 12(13)-EHC140-EF. Rockville, MD: Agency for Healthcare Research and Quality. 2012 www.effectivehealthcare.ahrq.gov/reports/ final.cfm. Consultado: 30/06/2020.

22. Ratcliffe L, Thomas W, Glen J, Padhi S, Pordes B, Wonderling D, et al. Diagnosis and management of iron deficiency in CKD: a summary of the NICE Guideline Recommendations and Their Rationale. Am J Kidney Dis. 2016;67(4):548-558.

23. Gluba-Brzózka A, Franczyk B, Olszewski R, Rysz J. The influence of inflammation on anemia in CKD patients. Int J Mol Sci 2020; 21(3):725; doi: 10.3390/ijms21030725

24. Tessitori N, Solero GP, Lippi G, Bassi A, Faccini GB, Bedogna V, et al. The role of iron status markers in predicting response to intravenous iron in haemodialysis patients on maintenance erythropoietin. Nephrol Dial Transplant. 2001;16(7):1416-23. doi: 10.1093/ndt

25. Bovy C, Tsobo C, Crapanzano L, Rorive G, Beguin Y, Albert A, et al. Factors determining the percentage of hypochromic red blood cells in hemodialysis patients. Kidney International. 1999;56:1113-1119.

26. Richardson D, Bartlett C, Will EJ. Optimizing erythropoietin therapy in hemodialysis patients. American Journal of Kidney Diseases. 2001;38(1):109-117. DOI: https://doi.org/10.1053/ajkd.2001.25203. Consultado el 13/10/2020.

27. Campistrús MN, Carlini RG, Andrade L, Chifflet L, Canziani ME, Obrador GT, et al. Encuesta sobre el control de la anemia en pacientes con enfermedad renal crónica en diálisis en Latinoamérica. Nefrol Latinoam 2018;15:16-28. www.nefrologialatinoamericana.com 